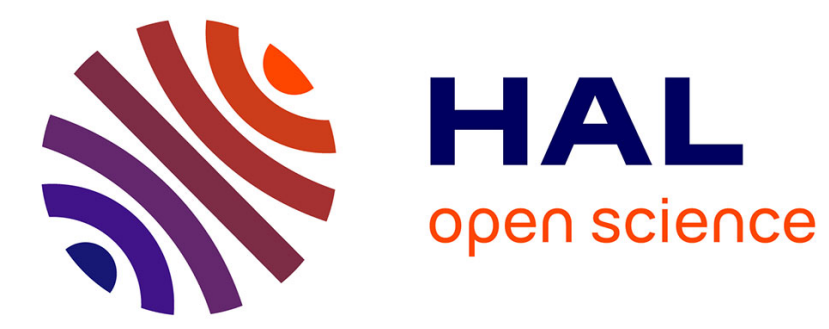

\title{
Split and flow: reconfigurable capillary connection for digital microfluidic
}

Florian Lapierre, Maxime Harnois, Yannick Coffinier, Rabah Boukherroub, V. Thomy

\section{- To cite this version:}

Florian Lapierre, Maxime Harnois, Yannick Coffinier, Rabah Boukherroub, V. Thomy. Split and flow : reconfigurable capillary connection for digital microfluidic. Lab on a Chip, 2014, 14, pp.3589-3593. 10.1039/C4LC00650J . hal-01058057

\section{HAL Id: hal-01058057 https://hal.science/hal-01058057}

Submitted on 18 Dec 2015

HAL is a multi-disciplinary open access archive for the deposit and dissemination of scientific research documents, whether they are published or not. The documents may come from teaching and research institutions in France or abroad, or from public or private research centers.
L'archive ouverte pluridisciplinaire HAL, est destinée au dépôt et à la diffusion de documents scientifiques de niveau recherche, publiés ou non, émanant des établissements d'enseignement et de recherche français ou étrangers, des laboratoires publics ou privés. 


\title{
Split and flow: reconfigurable capillary connection for digital mi- crofluidic
}

\author{
Florian Lapierre, ${ }^{a, b}$ Maxime Harnois, ${ }^{a, c}$ Yannick Coffinier $^{d}$, Rabah Boukherroub ${ }^{d}$ and Vincent \\ Thomy ${ }^{* a}$ \\ ${ }^{a}$ Institute of Electronics, Microelectronics and Nanotechnology (IEMN), UMR CNRS 8520, University Lille 1, F-59652 Villeneuve d'Ascq, France ${ }^{b}$ Micro/Nanophysics Research Laboratory, RMIT University,

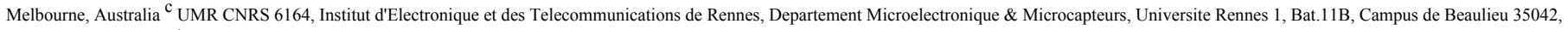 \\ Rennes Cedex, France ${ }^{\mathrm{d}}$ Interdisciplinary Research Institute (IRI), USR CNRS 3078, University Lille 1, 50 Avenue Halley, F-59658 Villeneuve d'Ascq, France
}

Liquid supplying to droplet-based microfluidic microsystems remains a delicate task facing the problems of coupling continuous to digital or macro to microfluidic systems. Here, we take advantage of superhydrophobic microgrids to address this problematic. Insertion of a capillary tube inside microgrid aperture leads to a simple and reconfigurable droplet generation set up.

\section{Introduction}

In the last twenty years, microfluidic systems have attracted impressive interest in the fields of biotechnology industries for healthcare monitoring, clinical diagnostics, chemical or biological reagents handling. ${ }^{1-4}$ These applications have common specificities and require new microsystems concepts for handling micro- to pico- volumes of reagents, detecting/analyzing at very high sensitivity rate, and sampling with minimal reagents consumption. To overcome these challenges, two main categories of microfluidic devices have been developed and can be classified as follow: Droplet-based MicroFluidics (DMF) and Continuous-based MicroFluidics $(\mathrm{CMF})$. DMF is usually based on liquid droplet, handled via electrowetting for instance. First introduced by Lippman 140 years ago ${ }^{5}$, Berge et al. demonstrated the benefit of a dielectric layer for reversible droplet actuation. ${ }^{6}$ As a result, moving, splitting and mixing of well-calibrated volume droplets could be easily performed with an electrode network. This multitasking microfluidics concept is nowadays one of the most used technologies for discrete liquid handling. ${ }^{7}$ However, loading samples into the device requires a "buffer tank", which constitutes one of the main drawbacks of DMF, espe-

$\dagger$ Electronic Supplementary Information (ESI) available: [details of any supplementary information available should be included here]. See DOI: 10.1039/b000000x/

a Institute of Electronics, Microelectronics and Nanotechnology (IEMN), UMR CNRS 8520, University Lille 1, F-59652 Villeneuve d'Ascq, France. E-mail: vincent.thomy@iemn.univ-lille1.fr

${ }^{b}$ School of Electrical and Computer Engineering, 394 Swanston Street, Melbourne VIC 3000 Australia.

c UMR CNRS 6164, Institut d'Electronique et des Telecommunications de Rennes, Departement Microelectronique \& Microcapteurs, Universite Rennes 1, Bat.11B, Campus de Beaulieu 35042, Rennes Cedex, France.

${ }^{d}$ Interdisciplinary Research Institute (IRI), USR CNRS 3078, University Lille 1, 50 Avenue Halley, F-59658 Villeneuve d'Ascq, France. cially for experiments that require large quantities of reagents and long operation times. The CMF technology uses welldefined microchannels for handling continuous flows of fluids in a closed device. Basic fluidic operations are accessible but require specific areas for each function. For instance, mixing can be generated by diffusion with or without turbulences ${ }^{8}$ splitting of continuous flow can be performed using non miscible fluids, but necessitates supplementary liquid access and additional liquid extraction step. ${ }^{9}$ The combination of the DMF and CMF techniques within the same device results in powerful microfluidic systems that ensure easy fluids handling (i.e. splitting, calibrating, mixing) thanks to DMF and easy transfer of high amount of fluid using CMF. An analogy can be made with Digital to Analog electronic converters for data handling. But in the case of fluid handling, the first limitation concerns the connection between the macro and micro worlds. This question is of particular interest for dropletbased devices where parameters such as droplet volume and speed have to be controlled. How to feed microsystem with liquid in a simple and reconfigurable manner?

Only a few recent studies deals with this concept. For instance, Kim et al. reported an Automated Digital Microfluidic system for DNA sequencing. ${ }^{10}$ In this device, DMF was used to operate repeated steps for library preparation and CMF capillaries interface was combined to allow easy fluid transfer between the two interfaces. Wheeler's group also demonstrated hybrid digital-channel microfluidics for pre-processing and chemical separations. ${ }^{11}$ Droplets of liquids are digitally displaced and mixed together prior to their loading in a PDMS channel by capillary action or electrokinetically. In 2010, the same group reported a modified architecture to interface a multilayer Digital-to-Channel design on glass. This configuration allowed droplets' dispensing from reservoirs and splitting droplets on chip. ${ }^{12,13}$ In the same context, Wu et al. described recently the fabrication of "Analog-to-Digital" and "Digitalto-Analog" (AD/DA) microfluidic converters. ${ }^{14}$ Using a series of microfabrication processes, they succeeded to integrate both CMF and DMF technologies in a single chip for liquidhandling and micro-analytical microsystems. All the systems reported in the literature describe the high potentialities of $\mathrm{CMF} / \mathrm{DMF}$ converters, but suffer from the lack of reconfigurability and adaptability. In fact, each CMF inlet and each 
outlet are fixed at a specific location near the DMF system. As a consequence it is impossible to reconfigure the system without redesigning and fabricating again the whole device.

From this statement, we propose in this paper an original solution to address the liquid supplying problem within a droplet-based microfluidic system. This strategy deals with an electrowetting set up where a water based droplet is squeezed between a surface composed of a hydrophobic electrode matrix and a counter-electrode. The key point of this work is the use of superhydrophobic microgrid, as counter-electrode, prepared using micro/nanofabrication techniques. Any aperture of this grid can be used to host a capillary, localized with the naked eye and fixed in a reversible manner authorizing a huge reconfigurability. Once the liquid flows continuously through the capillary tube, a droplet is easily formed by electrowetting; the superhydrophobic character of the grid avoids droplet pinning from the capillary. The technique developed herein has several advantages such as reconfigurability, flexibility, simplicity, and reduced time of assembly, compared to the reported techniques.

\section{Experimental}

\subsection{Reagents and materials}

In order to fabricate the ElectroWetting-On-Dielectric Digital Microfluidic (EW-DMF) lab-on-chip system and silicon nanowires substrates, clean room reagents including AZnLOF2070, AZ4562 and SU-8 2002 photoresist, MIF-326 and SU-8 developers from Micro Chemicals (Germany), hydrophobic Cytop from AGCCE (Netherlands), glass substrates from ACM Verre Industrie (France), and p-type $<100>$ crystalline highly doped silicon substrates $(0.0090 .01 \mathrm{ohm} / \mathrm{cm})$ from Siltronix (France), were used. Nickel deposition was performed with a PLASSYS MP 400S Electron Beam Evaporator (France). Hydrofluoric acid (50\%), hydrogen peroxide (30\%) were all purchased from Carlo Erba. Nitric acid (65\%) was purchased from Merck. Acetone, isopropyl alcohol ( $i$ $\mathrm{PrOH})$ were obtained from Sigma Aldrich. The continuous flow was controlled via the Fluigent Microfluidic Flow Control System (MFCS).

\subsection{Device fabrication}

A similar EW-DMF system as previously reported by Lapierre et al. ${ }^{15}$ and Jonsson et al. ${ }^{16}$ was used. The electrode network and final device are illustrated in Fig. 2 b. ${ }^{16}$ Briefly, $20 \mathrm{~nm}$ thick Ni layer was sputtered on a transparent glass substrate and the electrodes network was fabricated using standard photolithography. Then, $2 \mu \mathrm{m}$ of SU-8 2002 as dielectric layer followed by $30 \mathrm{~nm}$ of Cytop as hydrophobic layer were spin coated. This resulted in a contact angle and a contact angle

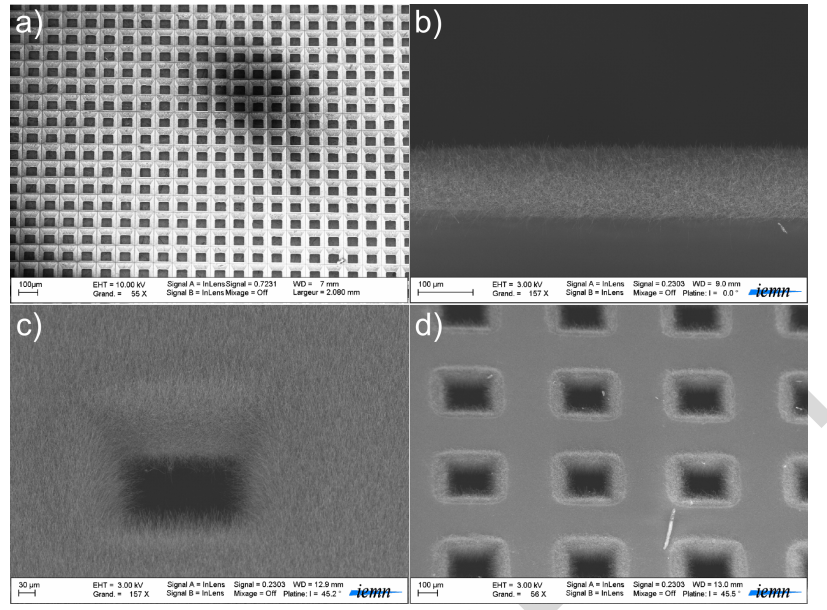

Fig. 1 SEM images of (a) the silicon grid in V-groove shape with $117 \mu m^{2}$ square holes at the top and $49 \mu m^{2}$ at the backside; (b) Silicon nanowires grown via VLS technique. This robust nanowires carpet is $50 \mu \mathrm{m}$ thick and ensures a complete superhydrophobic behaviour with Cassie-Baxter state; (c) Nanowires carpet surrounding one hole of the grid; (d) Backside of the grid with the presence of the nanowires carpet at the border of each hole. The backside hole was $250 \mu \mathrm{m}^{2}$ without nanowires and $\sim 150 \mu \mathrm{m}^{2}$ with the nanowire carpet.

hysteresis of $110^{\circ}$ and $11^{\circ}$, respectively. 96 electrodes placed on the base-plate are individually addressed and allow droplet displacement under EW.

The superhydrophobic microgrid counter-electrode was fabricated on a $380 \mu m$ thick, $<100>$ silicon wafer. First, a $200 \mathrm{~nm} S i_{x} N_{y}$ layer was deposited by LPCVD on both sides of the substrate. Optical lithography was used to produce squared areas (from $120 \mu \mathrm{m}$ to $250 \mu \mathrm{m}$ ). After removal of the photoresist, a $\mathrm{CHF}_{3} / \mathrm{CF}_{4}$ plasma was used to etch the silicon nitride exposing the square patterns. Silicon nitride was also removed on the backside. The substrate was then cleaned with acetone, ethanol and dried under nitrogen. Backside substrate was first thinned to $100 \mu \mathrm{m}$ in a $\mathrm{KOH}$ bath for $5 \mathrm{~h}$. Then, the grid was etched in the same bath; the $S i_{x} N_{y}$ mask protects the top wafer and only the $<111>$ oriented plan is etched at the naked squares. Anisotropic etching produced trapezoidal shape holes (Fig 1.a). A more detailed explanation of the fabrication process can be found in Brunet et al. ${ }^{17}$ In this study, the grids $(2 \mathrm{~cm} \times 2 \mathrm{~cm})$ with $250 \mu \mathrm{m}$ backside holes were selected. A $4 \mathrm{~nm}$ layer of Au was then sputtered on the top wafer. The metallized interface was introduced in a $500^{\circ} \mathrm{C} \mathrm{CVD} \mathrm{fur-}$ nace chamber under $\mathrm{SiH}_{4}$ flow. At this eutectic temperature, the gold film dewets to $10-100 \mathrm{~nm}$ nanodroplets which act as catalysts for silane decomposition. Due to oversaturation of the Au-Si droplets by silicon atoms, the formation of nuclei at the solid-liquid interface was obtained and nanowires elongated with the gold nanoparticle on top. Using $40 \mathrm{sccm}$ 
of $\mathrm{SiH}_{4}$, total pressure of $0.532 \mathrm{mbar}$ and growth time of 60 $\mathrm{min}$, silicon nanowires of $50 \mu \mathrm{m}$ in height were produced (Fig. 1.b). A more detailed description of the nanowires growth can be found in Brunet et al. ${ }^{18}$ and Lapierre et al. ${ }^{19}$ To achieve the superhydrophobic character of the counter-electrode, 30 $\mathrm{nm}$ thick $C_{4} F_{8}$ layer was deposited by plasma. The resulting contact angle and contact angle hysteresis were $160^{\circ}$ and $0^{\circ}$, respectively, evidencing the super-repellent property of the surface. The final microgrid surface displays on its top layer the nanowire carpet which covers entirely the trapezoidal holes (Fig. 1.c). On the backside surface, the presence of the nanowires is limited only to the border of each hole due to the inexistence of gold film on this side during the sputtering process. The optimal superhydrophobic (SH) microgrid selected for this experiment presented final hole opening of $\sim 150 \mu \mathrm{m}^{2}$ for the insertion of the capillary tubes.

Robustness of the SH microgrid with droplet impalement and liquid spreading was tested under drop impact experiment. Similar experimental bench described by Lapierre et al. ${ }^{19}$ allowed the measurement of the maximum dynamic pressure when the droplet passes through the SH microgrid. Video $1 \dagger$ shows that for a drop impact at $0.469 \mathrm{~m} / \mathrm{s}$, a little amount of liquid crossed the other side of the grid; the rest of the droplet bounced off the surface and was never impaled. The dynamical pressure can be calculated using $P_{e x t}=\rho U^{2}=247 P a$ with $\rho$, the density of the liquid equal to $1126 \mathrm{~kg} \cdot \mathrm{m}^{3}$ and $U$ the speed of the droplet before impact.

\section{Results and discussion}

The base-plate and SH microgrid counter-electrode were placed adjacent at $300 \mu m$ gap. The electrode network was controlled via a LabView routine and electrically supplied by a signal generator (CENTRAD GF 265, ELC, France) coupled with a $50 \mathrm{dBm}$ high-voltage amplifier (TEGAM, USA) set at $100 V_{T R M S} @ 1 \mathrm{kHz}$. A silica capillary tube $75 \mu \mathrm{m}$ inner diameter and $150 \mu \mathrm{m}$ outer diameter was inserted through one of the superhydrophobic holes of the grid and connected to the Fluigent MFCS (see Fig. 2). The nanowire carpet bound the capillaries and prevented them to detach from the microgrid. This is advantageous as no epoxy or silicon glue was required to seal each tube. It is to be noted that the capillary was always placed at the vicinity of the SH surface (on the top of the $\mathrm{V}$ groove hole) when inserted. The precise location of the capillary was performed in a reproducible manner by 3 methods of visualisation:

- Depth of the capillary was checked thanks to an horizontal line previously drawn on the capillary (view from the bottom of the system),

- Location of the capillary on the electrode network (view from the top, through the base plate), a)

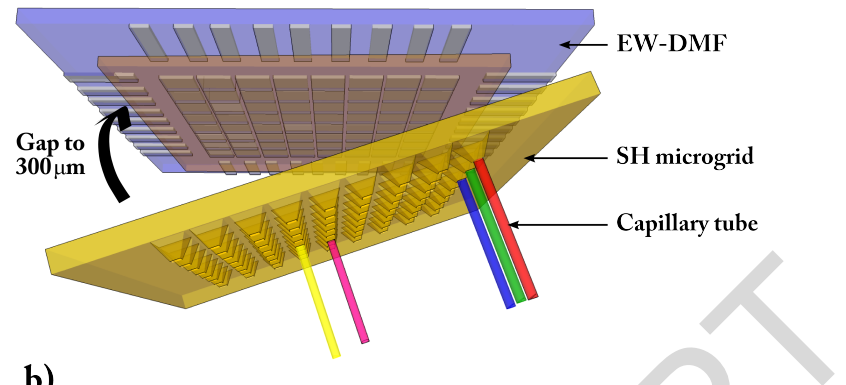

b)

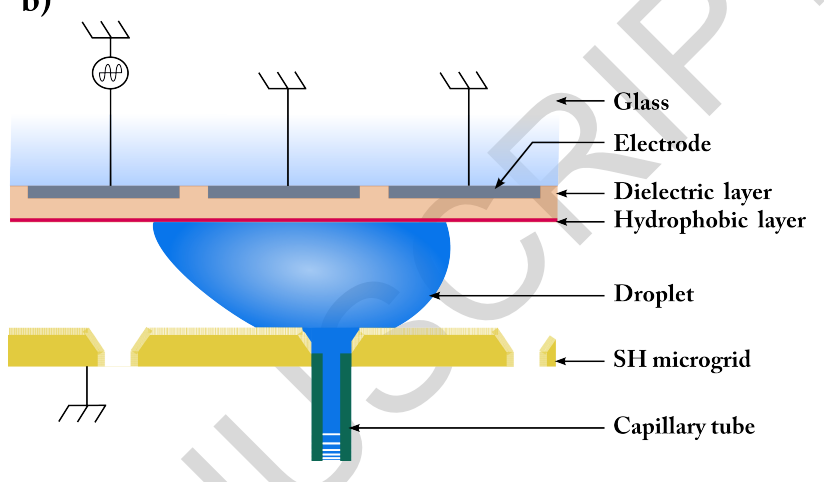

Fig. 2 (a) 3D view of the CMF/DMF microsystem. The SH microgrid acts as counter-electrode where tubing accesses are available on the whole EW-DMF electrode network. This setup allows easy reconfiguration of the microfluidic system for various applications. (b) Closer view of the EW-DMF coupled with a capillary tube. The base plate was composed of the transparent electrodes network, driving the droplet. The superhydrophobic microgrid $\left(\sim 150 \mu \mathrm{m}^{2}\right)$ counter-electrode allows capillary tube access $\left(150 \mu \mathrm{m}^{2}\right)$ without any use of epoxy or silicon rubber. The capillary tube can be located anywhere on the grid, depending on the user's need. The superhydrophobic character of the grid allows easy droplet detachment from the capillary, easy droplet motion and avoids leakage from the grid pores.

- Depth of the capillary with the view from the side of the microsystem,

Moreover, as the grid has a V-groove like shape, the nanowire carpet is protected from the insertion and removal of the capillary. Some of the nanowires at the bottom of the groove and backside of the silicon wafer could be destroyed by the capillary but this step does not affect the device functionalities. By selecting appropriate diameter, capillary tubes can be easily inserted into the grid just by eye sight, without any specific and complex tools such as microhandlers, and/or microscopes. Moreover capillaries can be relocated as desired along the electrode path. This major advantage allows extra reconfigurability of the lab-on-chip to allow various fluidic operations on demand, at any specific location.

A continuous water flow rate $(10 \mu L / \mathrm{min})$ was loaded into the EW-DMF system as shown in Fig. 3.a-b. As soon as the 


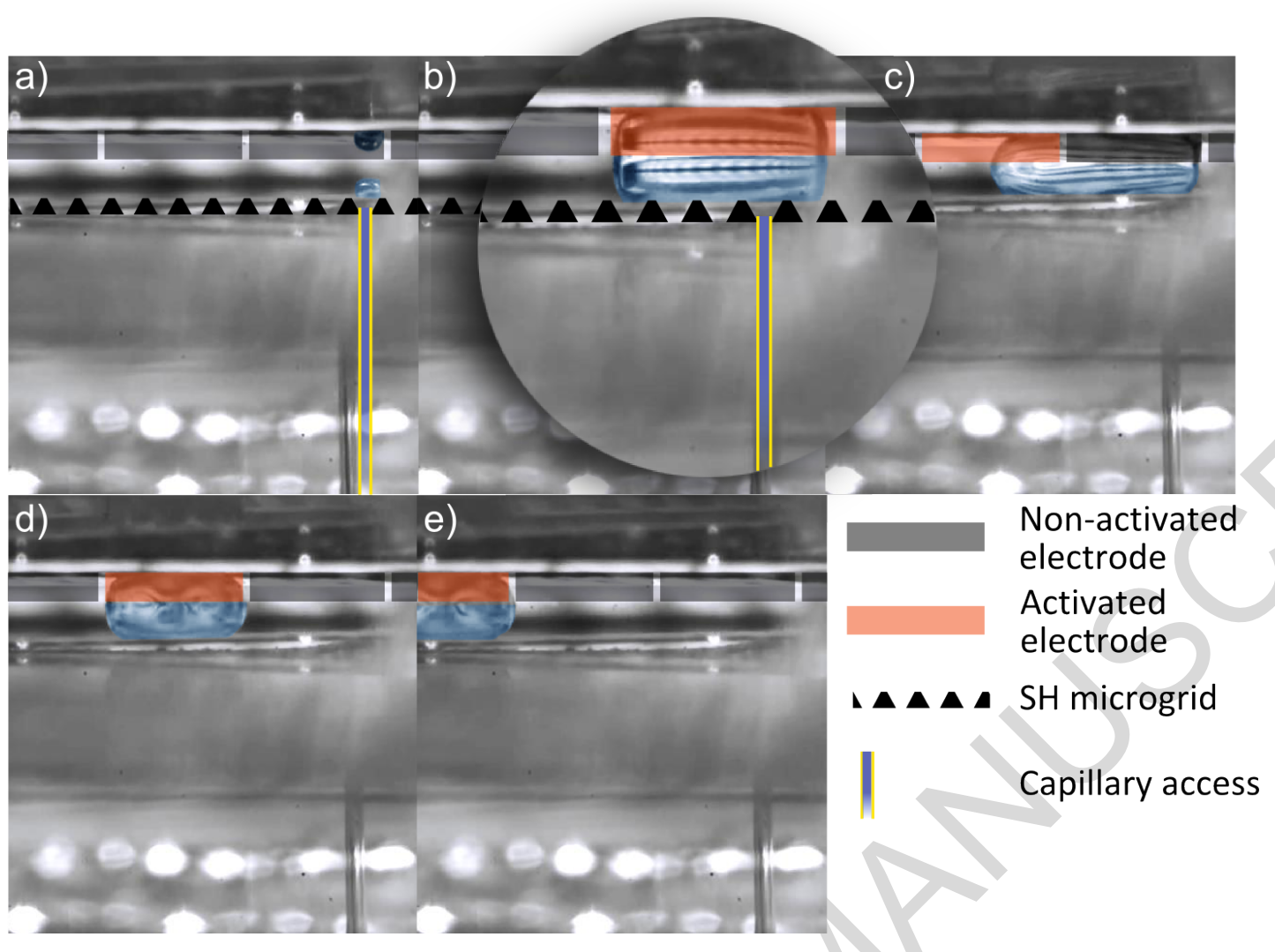

Fig. 3 Photographs of the CMF/DMF setup. (a-b) A $1 \mu L$ droplet is generated from the SH microgrid. (c-e) The DMF electrode network is sequentially activated to actuate the droplet. The grid holes are not "seen" by the droplet during its motion due to their SH character. No leakage is observed while generating the liquid droplet. Blue color was computationally added to differentiate the real droplet from its mirror on the base plate. Schematics of the grid, the electrode network and capillary were also added to help visualisation.

droplet volume reached $1 \mu L$ and the distance electrode/SH microgrid was adjusted to $300 \mu \mathrm{m}$, the electrode network was sequentially turned on in order to first, split the droplet from the tube and second, to move the droplet along the electrode network (Fig 3.c-e). Video $2 \dagger$, taken from the side of the microsystem, summarizes the fluidic sequence. The calibrated volume droplet was then available for other microfluidic tasks such as mixing, transporting, and merging also achieved by EW.

One of the most critical steps, the depinning of the droplet off the capillary, normally occurs during the droplet generation from the capillary. However, by using such a configuration, this challenge is overcome: the water repellency behaviour of the SH surface minimizes the wetted/sticky areas at the noozle part of the capillary. The liquid pinching mechanism is enhanced, leading to droplet splitting from the tubes. The high hole density allows multi-inlet/outlet accesses, but does not provoke liquid leakage out of the SH grid. Drop impact experiments showed that a dynamic external pressure of at least $250 \mathrm{~Pa}$ is necessary for the liquid to go through the $\mathrm{SH}$ grid. As the electrowetting is applied on the electrode base plate, no pressure is exerted on the SH surface. The droplet "sees" the holes as an air coffin where it can slide over. Furthermore, the superhydrophic property of the microgrid presents two crucial advantages regarding the aimed lab-on-chip applications. First, as detailed by Lapierre et al. ${ }^{15}$ and Jonsson et al. ${ }^{16}$, water repellent nanowire carpet ensures antibiofouling properties: biomolecules adsorbed non specifically on these surfaces can be easily removed by a simple water droplet motion that resulted in a very efficient lift-off of the bioparticles. Second, as shown by Nguyen et al. ${ }^{20}$, by using an appropriate surface chemistry, silicon nanowire surfaces presenting super repellent properties for a wide range of liquids (from $33 \mathrm{mN} / \mathrm{m}$ to $72 \mathrm{mN} / \mathrm{m}$ ) can be prepared. This is of prime interest for various applications knowing that the surface chemistry coatings are compatible with EW (no electrical field is applied to the counter-electrode, preserving the coating). By adding lateral walls on each side of the elec- 
trowetting electrodes, it is possible to fill this channel by oil. Due to the superomniphobic property of the micro-grid, this low surface tension liquid will be confined and biphasic electrowetting (water-based droplet in oil) should be easily realized. Moreover, by using these micro-grids coupled to a biphasic microfluidic setup, it should be possible to interact with water droplets without losing its sealing.

\section{Conclusion}

While connecting macroscopic world to microfluidic systems remains usually a question of experimenter patience and skills, we demonstrated in this work an original way to take advantage of the superhydrophobic microgrid interface to perform continuous to digital microfluidic operations. The technique proposed herein presents several advantages:

- Easy position of CMF capillaries at different locations on a microgrid. Capillaries can be located anywhere on the cover of the microfluidic system grid for reagent delivery into the digital microsystem.

- The use of a superhydrophobic grid is also advantageous as the capillary is fixed without requiring any glue or epoxy and it can be displaced reversibly from one grid aperture to the other.

- No leakage out of the microsystem occurs thanks to the liquid repellency behaviour of the SH microgrid. Furthermore, this solution can be employed for biphasic microfluidic authorizing droplet water based creation in an oil flux.

We do believe that the method developed in this work may represent a universal solution for coupling microfluidic microsystems to the macroscopic world.

\section{Acknowledgements}

The Centre National de la Recherche Scientifique (CNRS) and the European Community Seventh Frame-work Programme (FP7/ 2007-2013) under grant agreement no. 227243 are gratefully acknowledged for financial support.

\section{References}

1 G. M. Whitesides, Nature, 2006, 442, 368-373.

2 L. Y. Yeo, H.-C. Chang, P. P. Y. Chan and J. R. Friend, Small, 2011, 7, $12-48$.

3 P. N. Nge, C. I. Rogers and A. T. Woolley, Chem. Rev., 2013, 113, 25502583.

4 E. K. Sackmann, A. L. Fulton and D. J. Beebe, Nature, 2014, 507, 181189.

5 G. Lippmann, Ann. Chim. Phys., 1875, 5, 494-549.
6 B. Berge, C R Acad Sci IIb Mec, 1993, 317, 157-163.

7 M. J. Jebrail, M. S. Bartsch and K. D. Patel, Lab Chip, 2012, 12, $2452-$ 2463.

8 A. D. Stroock, S. K. W. Dertinger, A. Ajdari, I. Mezi, H. A. Stone and G. M. Whitesides, Science, 2002, 295, 647-651.

9 R. Seemann, M. Brinkmann, T. Pfohl and S. Herminghaus, Rep. Prog. Phys., 2012, 75, 016601.

10 H. Kim, M. J. Jebrail, A. Sinha, Z. W. Bent, O. D. Solberg, K. P. Williams, S. A. Langevin, R. F. Renzi, J. L. Van De Vreugde, R. J. Meagher, J. S. Schoeniger, T. W. Lane, S. S. Branda, M. S. Bartsch and K. D. Patel, PLoS ONE, 2013, 8, e68988.

11 M. Abdelgawad, M. W. L. Watson and A. R. Wheeler, Lab Chip, 2009, 9 , 1046-1051.

12 J. P. Rolland, R. M. Van Dam, D. A. Schorzman, S. R. Quake and J. M. DeSimone, J. Am. Chem. Soc., 2004, 126, 2322-2323.

13 E. Berthier, E. W. K. Young and D. Beebe, Lab Chip, 2012, 12, 1224 1237.

14 C. Wu, F. Bendriaa, F. Brunelle and V. Senez, Microelectron Eng, 2011, 88, 1878-1883.

15 F. Lapierre, G. Piret, H. Drobecq, O. Melnyk, Y. Coffinier, V. Thomy and R. Boukherroub, Lab Chip, 2011, 11, 1620-1628.

16 M. Jonsson-Niedziolka, F. Lapierre, Y. Coffinier, S. J. Parry, F. Zoueshtiagh, T. Foat, V. Thomy and R. Boukherroub, Lab Chip, 2011, 11, 490496.

17 P. Brunet, F. Lapierre, F. Zoueshtiagh, V. Thomy and A. Merlen, Appl. Phys. Lett., 2009, 95, 254102-3.

18 P. Brunet, F. Lapierre, V. Thomy, Y. Coffinier and R. Boukherroub, Langmuir, 2008, 24, 11203-11208.

19 F. Lapierre, P. Brunet, Y. Coffinier, V. Thomy, R. Blossey and R. Boukherroub, Farad. Discuss., 2010, 146, 125-139.

20 T. P. N. Nguyen, R. Boukherroub, V. Thomy and Y. Coffinier, J. Colloid Interface Sci., 2014, 416, 280-288. 